\title{
Return to Sport After Hip Arthroscopy for Femoroacetabular Impingement Syndrome: a Comprehensive Review of Qualitative Considerations
}

\author{
Sebastian J. Ko ${ }^{1}$ Michael A. Terry ${ }^{1} \cdot$ Vehniah K. Tjong $^{1}$ \\ Published online: 4 May 2020 \\ (C) Springer Science+Business Media, LLC, part of Springer Nature 2020
}

\begin{abstract}
Purpose of Review Arthroscopic hip surgery for femoroacetabular impingement syndrome has evolved over time and has resulted in significantly improved clinical outcomes. These outcomes can be measured by clinical and radiographic metrics. Return to sport is commonly used as an outcome measure, not only in terms of overall rate but also type of sport, level of competition, and timing of return, as its quantitative definition continues to develop. Qualitative research methods can highlight the patient-derived themes that affect an athlete's individual return to sport pathway, and can augment the existing methods of outcome reporting. We will specifically review the qualitative research that has been performed on evaluating return to sport after arthroscopic hip surgery for femoroacetabular impingement syndrome.

Recent Findings Current evidence finds a high overall rate of return to sport at 87-93\% after arthroscopic hip surgery for femoroacetabular impingement syndrome. The available qualitative research in this body of literature, which is limited, has found three main overarching themes behind athletes' decision and ability, or inability, to return to sport: self-efficacy, social support, and resetting expectations.

Summary Athletes experience high rates of return to sport and athletic performance after arthroscopic hip surgery for femoroacetabular impingement syndrome. This review highlights the qualitative considerations for these athletes in their overall readiness to return to sport, and its utility for treating physicians as we interact with these athletes both pre- and post-operatively. Further research is required to elucidate any further overarching themes that may be prevalent in different levels of competition.
\end{abstract}

Keywords Qualitative research · Qualitative assessment · Return to sport · Femoroacetabular impingement syndrome · Hip arthroscopy

\section{Introduction}

Femoroacetabular impingement syndrome (FAIS) is an increasingly recognized presentation of intra-articular hip pain corroborated by radiologic findings of pathoanatomy and can result in decreased performance in athletes involved in all levels of competition [1••]. It has also been shown to cause early-onset degenerative changes in young athletes, as early as

This article is part of the Topical Collection on Outcomes Research in Orthopedics

Vehniah K. Tjong

vehniah.tjong@northwestern.edu

1 Department of Orthopaedic Surgery, Northwestern University Feinberg School of Medicine, 259 East Erie Street, 13th Floor, Chicago, IL 60611, USA their second and third decades [2]. Biomechanically, it refers to the abnormal contact forces between impinging bone, and can originate from either the femoral side (CAM-type impingement), acetabular side (pincer-type impingement), or both (combined-type impingement). During hip range of motion, these forces can delaminate articular cartilage and damage the labrum [3].

Patients present with chief concerns of anterior hip pain radiating to the medial thigh, exacerbated by hip range of motion, prolonged periods in hip flexion (sitting), and extending the flexed hip against gravity and resistance (such as squatting, and getting in and out of a car) [4]. Pain can also present atypically in lateral or posterior locations around the hip [5]. The onset of pain is commonly gradual in nature, although some athletes may recall an acute precipitating episode. Symptoms are typically intermittent but may progress to being constant with ongoing chronic degeneration. Imaging 
evaluation involves standing radiographs including anteroposterior pelvis, false-profile, and lateral views. Advanced imaging modalities, including magnetic resonance imaging (with or without arthrography) and computed tomography can be utilized to gain three-dimensional appreciation of the underlying pathoanatomy, and assess for other possible diagnoses including stress fracture and avascular necrosis.

\section{Non-operative Management}

Non-operative treatment modalities include physical therapy, activity and lifestyle modifications, and oral medications. Given the relative low-risk profile and feasibility of these treatments during the season for athletes, these are often used as first line treatments in athletes presenting with FAIS, with recent evidence demonstrating improved clinical outcomes with non-operative treatment at 2 years [6]. Image-guided intra-articular injections are also used, for both diagnostic and therapeutic purposes. Local anesthetic can be infiltrated into the joint and, judging by the degree of pain relief, can inform both the athlete and treating physician how much of their pain is attributable to an intra-articular cause. Corticosteroids have traditionally been used for treatment effect, which has been shown to be variable in its intensity and duration. Benefit from these injections have been shown in a recent study to be a predictor of improved clinical outcomes after hip arthroscopy and should be included in the treatment armamentarium of treating physicians [7]. There have also been recent, though limited, studies on other injection treatments, including viscosupplementation (hyaluronic acid) and biologics (platelet-rich plasma, bone marrow aspirate, and stem-cell adjuncts). A recent systematic review found eight studies on 281 hips treated with injections, with findings suggesting that hyaluronic acid injections may be most effective at providing pain relief up to 12 months [8]. However, significant heterogeneity of pooled results and the small number of studies limits this recommendation, and injections continue to be an area of ongoing investigation.

\section{Operative Management and Outcomes}

Arthroscopic hip surgery has rapidly evolved, and is indicated for symptomatic patients presenting with FAIS and labral tears congruent with imaging findings. Compared with open and mini-open techniques, arthroscopic hip surgery has been shown to have superior results in health-related quality of life scores, along with fewer complications [9]. Early investigations showed modest outcomes, with one long-term outcome study with minimum follow-up of 10 years demonstrating $63 \%$ survivorship, with failure defined as conversion to total hip arthroplasty [10]. Notably, these patients were largely treated for their labral pathologies at that time, with the osseous anatomy ignored. Over time, as arthroscopic knowledge and techniques developed to include bony decompression and labral refixation, more successful clinical results and greater than $90 \%$ survivorship have been reported in the literature (Figs. 1 and 2) [7, 9, 11-20]. Improved outcomes are still seen in patients over the age of 40 , although these results may be tempered by a higher rate of conversion to total hip arthroplasty than in patients under 40 [20]. Investigations have also been aided by using more sophisticated clinical outcome reporting, such as patient-reported outcome measures (including the modified Harris Hip Score, International Hip Outcome Tool-12, Hip Outcome Score Sport-Specific Subscale) and patient satisfaction scores, in addition to using the minimal clinically important difference (MCID) and patient acceptable symptom state (PASS) for these scores. The quality, however, of literature reporting outcomes of surgical intervention for labral tears and FAI is still developing [19,21].

Radiographic results have also been reported, including comparison of pre- and post-operative $\alpha$-angles. However, multiple mid- and long-term outcome studies have shown not only no correlation between preoperative $\alpha$-angles and outcome scores but also no significant difference in clinical outcomes or satisfaction scores in athletes with a postoperative $\alpha$-angle of $<55^{\circ}$ as compared with $>55^{\circ}[13,15,22]$. Significant articular degenerative change (Tönnis grade II, $<$ $2 \mathrm{~mm}$ joint space) has been correlated to poorer clinical outcomes after hip arthroscopy, given that bony decompression of FAI may not adequately treat the pre-existing irreversible joint injury [23-25]. Byrd et al. recently found in their series of 200 consecutive athletes a trend towards worse clinical outcome scores with increasing Tönnis grade, although this did not reach statistical significance [26].

\section{Return to Sport After Hip Arthroscopy}

Return to sport (RTS) is also a commonly reported outcome metric used after arthroscopic hip surgery for FAIS. A recent systematic review on 31 studies involving 1981 hips among 1911 patients found a high rate of $87.7 \%$ of patients demonstrated RTS after surgery $(95 \%$ CI, $82.4-92.9 \%, p<0.001)$ [21]. The definition of return to sport continues to evolve from being evaluated as simply a binary outcome, to using multiple parameters including type of sport, level of competition, intensity level as compared with the pre-operative state, and frequency of athletic activity $[27,28,29 \bullet \bullet]$. Therefore, the RTS rate significantly depends on its definition and should be interpreted in the literature with care given this heterogeneity. Indeed, trends of lower return to sport have been reported in athletes involved in contact versus noncontact sports [27]. A recent study evaluating 131 professional athletes across four major North American team sports who underwent 


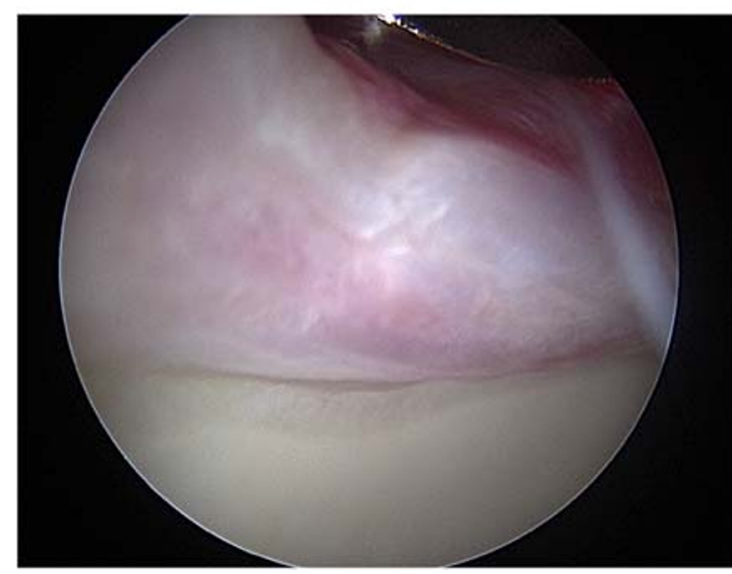

Fig. 1 Arthroscopic view of typical anterosuperior labral tear

151 arthroscopic hip surgeries showed an overall RTS rate of $88.7 \%$, with no significant difference in this overall rate between sports. However, the authors also found that the MLB and NHL athletes experienced a decrease in games played in the first season following hip arthroscopy $(p=0.04, p=0.01$, respectively), and NHL athletes also experienced a decreased in games played in seasons 2 and 3 postoperatively ( $p=$ 0.001 ), accompanied with a decrease in performance scores. Rehabilitation protocols after arthroscopic hip surgery for FAIS also lack standardization across institutions. O'Connor et al. conducted a systematic review on the RTS protocols and functional assessment after hip arthroscopy, and found that only two of 22 studies satisfied the criteria for a sufficient RTS protocol, and three of 22 provided a specific replicable test for RTS. Furthermore, Memon et al. showed in their systematic review on FAIS RTS studies that the criteria for RTS in $64 \%$ of the studies were simply completion of the rehabilitation protocol. Indeed, despite a high overall rate of RTS and improvement in patient-reported outcomes after hip arthroscopy, there is a lack of consensus in the literature regarding its objective definition.

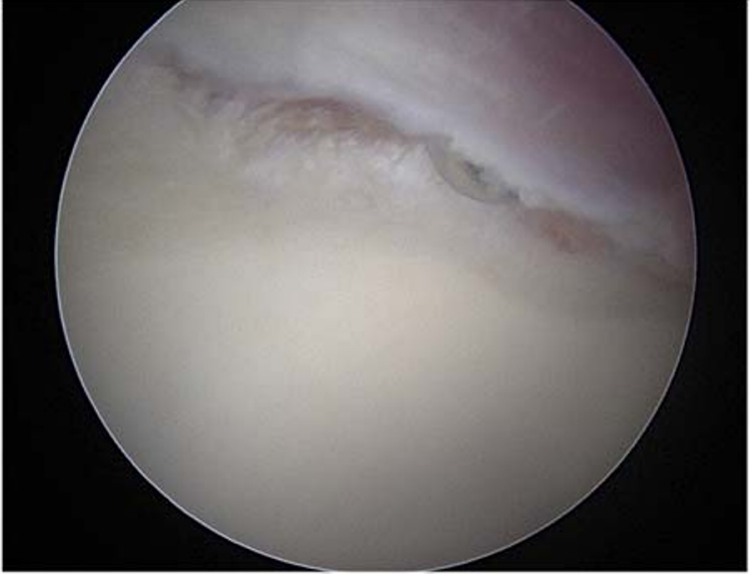

\section{Return to Sport After Hip Arthroscopy-Qualitative Considerations}

In addition to more quantitative measures of RTS that have been reported including overall rate, timing, and seasons played, qualitative research has recently been an area of focus to assess an athlete's readiness to return to their sport and activity level. Patient-derived concepts and themes which affect the RTS process can be identified through semi-structured interviews using open-ended questions from previous reviews of qualitative studies pertaining to athletic injury [30, 31]. Intrinsic and extrinsic factors such as competing interests, kinesiophobia, age, and internal stressors, and motivators can have a major effect on an athlete's decision to return to sport [32]. Fear of re-injury and loss of confidence have been shown to significantly affect an athlete's readiness to return to sport, regardless of their physical state [33-35]. Similarly, anxiety and depression, along with maladaptive coping methods including venting and denial can predict worse outcomes of not only post-operative rehabilitation and recovery but also may ultimately affect the decision to return to sport

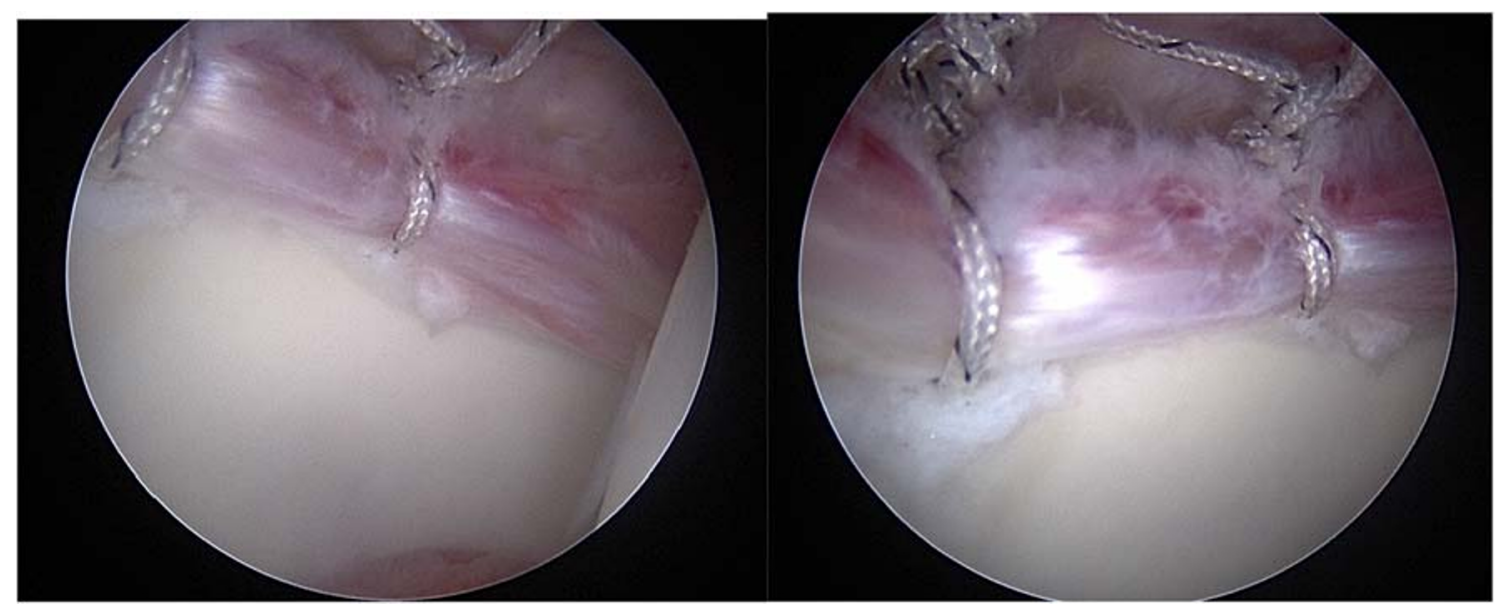

Fig. 2 Arthroscopic view after bony decompression with acetabuloplasty and labral repair with suture anchors 
itself. Christino et al. highlighted how self-efficacy can have as equal an impact as knee stability in return to sport after ACL reconstruction [36]. Evaluating these traits and their impact on the RTS process after surgery can better inform treating physicians in the preoperative discussion with these athletes. Recent literature has investigated RTS after anterior cruciate ligament reconstruction and arthroscopic Bankart repair using qualitative methods, which found overarching themes that were instrumental in RTS, such as fear of re-injury, shifts in priority, lifestyle changes, and innate personality traits $[34,35]$. Elements of these factors were shown to largely influence the decision to return to the pre-injury level sport not only in those patients who had returned but also those who had not returned to sport, more than other factors including the surgeon's advice not to return.

Recent qualitative research conducted on RTS after arthroscopic hip surgery found significant correlation between certain adaptive and maladaptive coping mechanisms and return to sport, and elucidated three distinct overarching themes: level of self-efficacy, social support, and resetting expectations (Table 1) [37••]. Firstly, in terms of self-efficacy, athletes who returned to their pre-injury level of activity cited their intrinsic motivated personality traits as the main factor leading to their successful recovery. Self-efficacy can be defined as "the belief in one's ability to succeed and persevere through challenges and maintain commitment to a cause" [36]. Examples of such responses included "focused on the 1 goal of playing again", "mind over matter", and even "I've been clean for 13 years now and on the road since, hence my sheer motivation to get back as soon as I could". These highlight the active coping mechanisms in those who were able to return to their sport after hip arthroscopy for FAIS, which has also been shown in the ACL population.

Secondly, social support is an extrinsic factor that was also prevalent in the RTS process after hip arthroscopy. This included support from the surgical team, therapy teams, family, and teammates. Examples of such responses included "going to PT became almost social for me and helped me to re-engage in a team-like atmosphere", "he was like a strict school teacher with clear expectations at certain time points", and "good rapport with my surgeon allowed me to trust him and feel confident in taking steps forward". These patients coped with these strategies of emotional connection in their postoperative recovery and eventual RTS, and use this sense of support as a buffer to stress from both injury and surgery. Importantly, the lack of support and feelings of isolation have been highlighted as two of the most common problems facing some athletes in the postoperative phase, and is confirmed in those athletes undergoing hip arthroscopy for FAIS [37, 38]. The concept of sharing the burden benefits these patients and provides a healthy environment that may foster eventual return to sport.

Thirdly, resetting expectations is another adaptive strategy. This was interestingly most relevant to those athletes who did not return to their pre-injury level of sport but still experienced good clinical outcome. Examples of such responses included "I know that my hip won't ever feel like it's 20 again, but that doesn't stop me from being active - I've stopped running but I picked up swimming", just because I'm not in college anymore, does not mean I have to stop skiing - I know I cannot be as competitive anymore, and that's OK". These patients can be thought of as "copers", or patients who are known to undergo and overcome transition points and minor setbacks,
Table 1 Reliance on adaptive and maladaptive coping strategies

\begin{tabular}{lll}
\hline & $\begin{array}{l}\text { Patients who returned } \\
\text { to pre-injury sport }\end{array}$ & $\begin{array}{l}\text { Patients who did not } \\
\text { return to pre-injury sport }\end{array}$ \\
\hline $\begin{array}{ll}\text { Adaptive mechanisms } \\
\text { Active coping }\end{array}$ & 9 & 2 \\
Acceptance & 5 & 5 \\
Reframing & 7 & 1 \\
Planning & 8 & 0 \\
Humor & 4 & 4 \\
Instrumental support & 4 & 3 \\
Self-distraction & 4 & 2 \\
Emotional support & 4 & 1 \\
Positive religion & 2 & 0 \\
Maladaptive mechanisms & & 1 \\
Venting & 1 & 1 \\
Self-blame & 0 & 1 \\
Behavioral disengagement & 0 & 0 \\
Substance abuse & 0 & 0 \\
Denial & 0 & \\
\hline
\end{tabular}


leading to higher global quality of life scores, and higher likelihood to return to sport, as demonstrated in those patients who underwent ACL reconstruction [39-41]. Adaptive strategies such as acceptance and reframing to set new activity expectations and limitations after arthroscopic hip surgery are the main factors in this theme.

Of the 23 athletes interviewed after hip arthroscopy, 13 $(57 \%)$ were able to return to their pre-injury sport level, whereas 10 (435) did not. This seemingly low RTS rate highlights its often ambiguous definition in the literature, since only those patients who had identical pre-injury and postoperative values in the type of sport, level of competition, and frequency of activity were classified in the study as successfully returning to sport. Furthermore, all but one of the patients in the study participated at a recreational level, whereas higher-level athletes have been demonstrated to have superior clinical outcomes. This was highlighted in a recent study comparing outcomes of elite athletes (professional, semiprofessional, or collegiate) and non-athletes after hip arthroscopy for treatment of FAIS, where elite athletes attain superior 2-year patient-reported outcomes and achieve MCID and PASS at higher rates when compared with non-athletes [18]. This discrepancy in findings between levels of athletes support the qualitative research findings discussed above, given it is well known that professional athletes possess the trait of self-efficacy in order to perform at a high level, which may also be influenced by financial gains [42]. High level athletes may also have improved access to health care providers, which may explain the overarching theme of social support in the RTS process. Mitchell et al. suggest that an athlete's perceived level of social support closely correlates with a player's self-esteem, emotional stability, and feelings of belonging [38]. Furthermore, a case-control study evaluating postoperative pain after arthroscopic hip surgery found that mental health history screening positive for anxiety and depression was an independent predictor [43]. Evidently, the overarching themes found through qualitative methods in in this patient population correlate well with the findings in the recent overall literature on outcomes and RTS after arthroscopic hip surgery.

Interestingly, the themes of fear of re-injury and kinesiophobia linked to injury flashbacks was found to be less instrumental in patients after hip arthroscopy for FAIS compared with those who underwent ACL reconstruction and Bankart repair. This is perhaps due to the traumatic nature of ACL ruptures and acute shoulder labral tears, as compared with the cumulative trauma more typically seen with FAIS.

\section{Conclusion}

FAIS is a condition now commonly seen in athletes with recent improvements in clinical examination, diagnostic imaging, and overall awareness. Results after arthroscopic hip surgery have evolved as techniques have developed, with improved clinical outcome scores and high rates of return to sport. However, as several recent systematic reviews have shown, the quality of reporting outcomes is still improving, and the definition of return to sport needs to be scrutinized for each study [21, 44]. Radiographic parameters, including Tönnis grade and comparison of pre- and post-operative $\alpha$ angles, have been called into question as predictors and measures of outcome [13, 22, 26]. Qualitative research methods offer insight into the psychological readiness to return to sport in athletes, and has shown overarching themes of self-efficacy, social support, and resetting expectations in athletes undergoing arthroscopic surgery for FAIS as they progress along their individual pathways to pre-injury level of sport. This research method augments the existing literature on return to sport after hip arthroscopy for FAIS, and its findings can be used to build rapport with athletes in preoperative discussions and postoperative rehabilitation.

\section{Compliance with Ethical Standards}

Conflict of Interest All authors report no conflicts of interest.

Human and Animal Rights and Informed Consent This article does not contain any studies with human or animal subjects performed by any of the authors.

\section{References}

Papers of particular interest, published recently, have been highlighted as:

- Of importance

- Of major importance

1.• Griffin DR, Dickenson EJ, O’Donnell J, et al. The Warwick Agreement on femoroacetabular impingement syndrome (FAI syndrome): an international consensus statement. Br J Sports Med. 2016;50(19):1169-76. https://doi.org/10.1136/bjsports-2016096743 Consensus statement from an international panel of 22 expert clinicians and academics regarding the key terminology, diagnosis, and treatment related to FAIS.

2. Byrd JWT. Femoroacetabular impingement in athletes, part 1. Sport Heal A Multidiscip Approach. 2010;2(4):321-33. https://doi.org/ 10.1177/1941738110368392.

3. Zadpoor AA. Etiology of femoroacetabular impingement in athletes: a review of recent findings. Sport Med. 2015;45(8):1097106. https://doi.org/10.1007/s40279-015-0339-2.

4. Philippon MJ, Schenker ML. Arthroscopy for the treatment of femoroacetabular impingement in the athlete. Clin Sports Med. 2006;25(2):299-308. https://doi.org/10.1016/j.csm.2005.12.006.

5. Clohisy JC, Knaus ER, Hunt DM, Lesher JM, Harris-Hayes M, Prather H. Clinical presentation of patients with symptomatic anterior hip impingement. In: Clinical Orthopaedics and Related Research. Vol 467. Springer New York. 2009, pp 638-644. https://doi.org/10.1007/s11999-008-0680-y 
6. Pennock AT, Bomar JD, Johnson KP, Randich K, Upasani VV. Nonoperative management of femoroacetabular impingement: a prospective study. Am J Sports Med. 2018;46(14):3415-22. https://doi.org/10.1177/0363546518804805.

7. Sogbein OA, Shah A, Kay J, Memon M, Simunovic N, Belzile EL, et al. Predictors of outcomes after hip arthroscopic surgery for femoroacetabular impingement: a systematic review. Orthop J Sport Med. 2019;7(6):2325967119848982. https://doi.org/10. $1177 / 2325967119848982$.

8. Khan W, Khan M, Alradwan H, Williams R, Simunovic N, Ayeni OR. Utility of intra-articular hip injections for Femoroacetabular impingement: a systematic review. Orthop J Sport Med. 2015;3(9):2325967115601030. https://doi.org/10.1177/ 2325967115601030 .

9. Nwachukwu BU, Rebolledo BJ, McCormick F, Rosas S, Harris JD, Kelly BT. Arthroscopic versus open treatment of femoroacetabular impingement: a systematic review of medium- to long-term outcomes. Am J Sports Med. 2016;44(4):1062-8. https://doi.org/10. $1177 / 0363546515587719$.

10. McCarthy JC, Jarrett BT, Ojeifo O, Lee JA, Bragdon CR. What factors influence long-term survivorship after hip arthroscopy? Clin Orthop Relat Res. 2011;469(2):362-71. https://doi.org/10. 1007/s11999-010-1559-2.

11. Gohal C, Shamshoon S, Memon M, Kay J, Simunovic N, Randelli F, et al. Health-related quality of life after hip arthroscopy for femoroacetabular impingement: a systematic review and meta-analysis. Sports Health. 2019;11(3):209-17. https://doi.org/10.1177/ 1941738119838799.

12. de Sa D, Lian J, Sheean AJ, et al. A systematic summary of systematic reviews on the topic of hip arthroscopic surgery. Orthop J Sport Med. 2018;6(9):1-12. https://doi.org/10.1177/ 2325967118796222

13. Tjong VK, Gombera MM, Kahlenberg CA, Patel RM, Han B, Deshmane $\mathrm{P}$, et al. Isolated acetabuloplasty and Labral repair for combined-type femoroacetabular impingement: are we doing too much? Arthrosc - J Arthrosc Relat Surg. 2017;33(4):773-9. https:// doi.org/10.1016/j.arthro.2016.10.022.

14. Nho SJ, Magennis EM, Singh CK, Kelly BT. Outcomes after the arthroscopic treatment of femoroacetabular impingement in a mixed group of high-level athletes. Am J Sports Med. 2011;39(1_supp1):14-9. https://doi.org/10.1177/ 0363546511401900 .

15. Philippon MJ, Weiss DR, Kuppersmith DA, Briggs KK, Hay CJ. Arthroscopic labral repair and treatment of femoroacetabular impingement in professional hockey players. Am J Sports Med. 2010;38(1):99-104. https://doi.org/10.1177/0363546509346393.

16. Byrd JWT, Jones KS. Hip arthroscopy in athletes: 10-year followup. Am J Sports Med. 2009;37(11):2140-3. https://doi.org/10. $1177 / 0363546509337705$.

17. Amenabar T, O'Donnell J. Return to sport in Australian football league footballers after hip arthroscopy and midterm outcome. Arthrosc - J Arthrosc Relat Surg. 2013;29(7):1188-94. https://doi. org/10.1016/j.arthro.2013.05.001.

18. Clapp IM, Nwachukwu BU, Beck EC, Jan K, Gowd AK, Nho SJ. Comparing outcomes of competitive athletes versus nonathletes undergoing hip arthroscopy for treatment of femoroacetabular impingement syndrome. Am J Sports Med. 2020;48(1):159-66. https://doi.org/10.1177/0363546519885359.

19. Bedi A, Chen N, Robertson W, Kelly BT. The management of labral tears and femoroacetabular impingement of the hip in the young, active patient. Arthrosc - J Arthrosc Relat Surg. 2008;24(10):1135-45. https://doi.org/10.1016/j.arthro.2008.06. 001.

20. Horner NS, Ekhtiari S, Simunovic N, Safran MR, Philippon MJ, Ayeni OR. Hip arthroscopy in patients age 40 or older: a systematic review. Arthrosc - J Arthrosc Relat Surg. 2017;33(2):464-475.e3. https://doi.org/10.1016/j.arthro.2016.06.044.

21. Minkara AA, Westermann RW, Rosneck J, Lynch TS. Systematic review and meta-analysis of outcomes after hip arthroscopy in femoroacetabular impingement. Am J Sports Med. 2019;47(2): 488-500. https://doi.org/10.1177/0363546517749475.

22. Bhatia S, Briggs K, Philippon M, Soares E. Post-operative alpha angle not associated with outcomes 5 years following hip arthroscopy for FAI. Arthrosc J Arthrosc Relat Surg. 2016;32(6):e12-3. https://doi.org/10.1016/j.arthro.2016.03.060.

23. Domb BG, Gui C, Lodhia P. How much arthritis is too much for hip arthroscopy: a systematic review. Arthroscopy. 2015;31(3):520-9. https://doi.org/10.1016/j.arthro.2014.11.008.

24. Gomberawalla MM, Kelly BT, Bedi A. Interventions for hip pain in the maturing athlete: the role of hip arthroscopy? Sports Health. 2014;6(1):70-7. https://doi.org/10.1177/1941738113497678.

25. Shah A, Kay J, Memon M, et al. Clinical and radiographic predictors of failed hip arthroscopy in the management of dysplasia: a systematic review and proposal for classification. Knee Surg Sport Traumatol Arthrosc. 2019;0(0):0. https://doi.org/10.1007/ s00167-019-05416-3

26. Byrd JWT, Jones KS, Bardowski EA. Influence of Tönnis grade on outcomes of arthroscopy for FAI in athletes: a comparative analysis. J Hip Preserv Surg. 2018;5(2):162-5. https://doi.org/10.1093/ JHPS/HNY011.

27. Ishøi L, Thorborg K, Kraemer O, Hölmich P. Return to sport and performance after hip arthroscopy for femoroacetabular impingement in 18- to 30-year-old athletes: a cross-sectional cohort study of 189 athletes. Am J Sports Med. 2018;46(11):2578-87. https://doi. org $/ 10.1177 / 0363546518789070$

28. Wörner T, Thorborg K, Stålman A, Webster KE, Momatz Olsson H, Eek F. High or low return to sport rates following hip arthroscopy is a matter of definition? Br J Sports Med. 2018;52(22):1475-6. https://doi.org/10.1136/bjsports-2018-099154.

29.• Ardern CL, Glasgow P, Schneiders A, et al. 2016 Consensus statement on return to sport from the First World Congress in Sports Physical Therapy. Bern. Br J Sports Med. 2016;50(14):853-64. https://doi.org/10.1136/bjsports-2016-096278 Consensus statement from an international panel of 17 expert clinicians and academics regarding the decision-making for return to sport and how it is currently seen as a continuum, as opposed to a binary outcome.

30. Malterud K. Qualitative research: standards, challenges, and guidelines. Lancet. 2001;358(9280):483-8. https://doi.org/10.1016/ S0140-6736(01)05627-6.

31. Marshall MN. Sampling for qualitative research. Fam Pract. 1996;13(6):522-5. https://doi.org/10.1093/fampra/13.6.522.

32. Clement D, Arvinen-Barrow M, Fetty T. Psychosocial responses during different phases of sport-injury rehabilitation: a qualitative study. J Athl Train. 2015;50(1):95-104. https://doi.org/10.4085/ 1062-6050-49.3.52.

33. Ardern CL, Österberg A, Tagesson S, Gauffin H, Webster KE, Kvist J. The impact of psychological readiness to return to sport and recreational activities after anterior cruciate ligament reconstruction. Br J Sports Med. 2014;48(22):1613-9. https://doi.org/ 10.1136/bjsports-2014-093842.

34. Tjong VK, Murnaghan ML, Nyhof-Young JM, Ogilvie-Harris DJ. A qualitative investigation of the decision to return to sport after anterior cruciate ligament reconstruction: to play or not to play. Am J Sports Med. 2014;42(2):336-42. https://doi.org/10.1177/ 0363546513508762.

35. Tjong VK, Devitt BM, Murnaghan ML, Ogilvie-Harris DJ, Theodoropoulos JS. A qualitative investigation of return to sport after arthroscopic Bankart repair: beyond stability. Am J Sports Med. 2015;43(8):2005-11. https://doi.org/10.1177/ 0363546515590222 . 
36. Christino MA, Fantry AJ, Vopat BG. Psychological aspects of recovery following anterior cruciate ligament reconstruction. J Am Acad Orthop Surg. 2015;23(8):501-9. https://doi.org/10.5435/ JAAOS-D-14-00173.

37.• Tjong VK, Cogan CJ, Riederman BD, Terry MA. A qualitative assessment of return to sport after hip arthroscopy for femoroacetabular impingement. Orthop J Sport Med. 2016;4(11): 1-7. https://doi.org/10.1177/2325967116671940 First salient article using qualitative methods to assess return to sport in athletes after arthroscopic hip surgery for FAIS. This research found three overarching themes directly correlating with return to sport: self efficacy, social support, and resetting expectations.

38. Mitchell I, Evans L, Rees T, Hardy L. Stressors, social support, and tests of the buffering hypothesis: effects on psychological responses of injured athletes. Br J Health Psychol. 2014;19(3):486-508. https://doi.org/10.1111/bjhp.12046.

39. Chmielewski TL, Hurd WJ, Snyder-Mackler L. Elucidation of a potentially destabilizing control strategy in ACL deficient noncopers. J Electromyogr Kinesiol. 2005;15(1):83-92. https://doi. org/10.1016/j.jelekin.2004.07.003XY.

40. Kaplan Y. Identifying individuals with an anterior cruciate ligament-deficient knee as copers and noncopers: a narrative literature review. J Orthop Sports Phys Ther. 2011;41(10):75866. https://doi.org/10.2519/jospt.2011.3384.

41. Schilaty ND, Nagelli C, Hewett TE. Use of objective neurocognitive measures to assess the psychological states that influence return to sport following injury. Sport Med. 2016;46(3): 299-303. https://doi.org/10.1007/s40279-015-0435-3.

42. Podlog L, Eklund RC. Returning to competition after a serious injury: the role of self-determination. J Sports Sci. 2010;28(8): 819-31. https://doi.org/10.1080/02640411003792729.

43. Stone AV, Malloy P, Beck EC, Neal WH, Waterman BR, BushJoseph CA, et al. Predictors of persistent postoperative pain at minimum 2 years after arthroscopic treatment of femoroacetabular impingement. Am J Sports Med. 2019;47(3):552-9. https://doi.org/ $10.1177 / 0363546518817538$.

44. O'Connor M, Minkara AA, Westermann RW, Rosneck J, Lynch TS. Return to play after hip arthroscopy: a systematic review and meta-analysis. Am J Sports Med. 2018;46(11):2780-8. https://doi. org/10.1177/0363546518759731.

Publisher's note Springer Nature remains neutral with regard to jurisdictional claims in published maps and institutional affiliations. 